\title{
A Vector Field Approach to Estimating Environmental Exposure Using Human Activity Data
}

\author{
Zijian Guo $^{1}{ }^{1}$, Xintao Liu ${ }^{1,2, *} \mathbb{1}$ and Pengxiang Zhao ${ }^{3}$ \\ 1 Department of Land Surveying and Geo-Informatics, The Hong Kong Polytechnic University, Hong Kong; \\ zijian1.guo@connect.polyu.hk \\ 2 Smart Cities Research Institute, The Hong Kong Polytechnic University, Hong Kong \\ 3 GIS Center, Department of Physical Geography and Ecosystem Science, Lund University, \\ 22362 Lund, Sweden; pengxiang.zhao@nateko.lu.se \\ * Correspondence: xintao.liu@polyu.edu.hk; Tel.: +852-2766-5962
}

Citation: Guo, Z.; Liu, X.; Zhao, P. A Vector Field Approach to Estimating Environmental Exposure Using Human Activity Data. ISPRS Int. J. Geo-Inf. 2022, 11, 135. https:// doi.org/10.3390/ijgi11020135

Academic Editor: Wolfgang Kainz

Received: 19 December 2021

Accepted: 12 February 2022

Published: 15 February 2022

Publisher's Note: MDPI stays neutral with regard to jurisdictional claims in published maps and institutional affiliations.

Copyright: (C) 2022 by the authors. Licensee MDPI, Basel, Switzerland. This article is an open access article distributed under the terms and conditions of the Creative Commons Attribution (CC BY) license (https:// creativecommons.org/licenses/by/ $4.0 /)$.

\begin{abstract}
Environmental exposure of people plays an important role in assessing the quality of human life. The most existing methods that estimate the environmental exposure either focus on the individual level or do not consider human mobility. This paper adopts a vector field generated from the observed locations of human activities to model the environmental exposure at the population level. An improved vector-field-generation method was developed by considering people's decisionmaking factors, and we proposed two indicators, i.e., the total exposure indicator (TEI) and the average exposure indicator (AEI), to assess various social groups' environmental exposure. A case study about the risky environmental exposure of coronavirus disease 2019 (COVID-19) was conducted in Guangzhou, China. Over 900 participants with various socioeconomic backgrounds were involved in the questionnaire, and the survey-based activity locations were extracted to generate the vector field using the improved method. COVID-19 pandemic exposure (or risk) was estimated for different social groups. The findings show that people in the low-income group have an $8 \%$ to $10 \%$ higher risk than those in the high-income group. This new method of vector field may benefit geographers and urban researchers, as it provides opportunities to integrate human activities into the metrics of pandemic risk, spatial justice, and other environmental exposures.
\end{abstract}

Keywords: vector field; mobility pattern; environmental exposure; spatial justice

\section{Introduction}

Environmental exposure is associated with human health and has attracted the attention of researchers [1,2]. The environment can be classified into two categories, i.e., a healthy environment and a harmful one. Exposure to harmful environments is a risk to people's health. However, different social groups are exposed to different risks when facing an environmental crisis [3,4]. Revealing this injustice is one of the important significances of studying environmental exposure. To investigate two social groups' disparity, we needed to quantify the environmental exposure first, and then do a comparative analysis between different social groups. However, environmental conditions may not be the only factor causing inequity. As Soja indicates in spatial justice [5], the current urban system's mechanism results in the unfair redistribution of social benefits, tilting more to the rich than the poor. About the mechanism, Soja argues that two social groups' accumulation of locational decisions (ALD) plays an important role. Thus, when quantifying environmental exposure, we should take ALD into account, which involves three key elements: (1) quantifying the exposure at an aggregated level; (2) measuring with spatial properties; and (3) reflecting people's decisions. Some previous studies have attempted to quantify the exposure, but few perfectly capture these three elements at the same time, e.g., [6-9]. Some researchers have studied the aggregated level's exposure using the demographic data [10-13]. However, these studies do not take people's mobility and spatial interaction 
into consideration. Yang et al.'s [14] study investigates delivery men's individual activity space and reveals the spatial unbalance of the environmental exposure in aspects of air pollution and noise pollution. However, their study lacks an aggregated level perspective, and it is hard to evaluate different social groups' disparity. The vector field method has the advantage of fully considering mobility from a population perspective [15-17]. However, it still lacks the third element, i.e., people's decisions, and furthermore, this method is rarely applied in environmental exposure studies.

Motivated by the gaps mentioned above, this paper develops an improved vectorfield method by considering crowd decision-making. Based on the improved method, we propose a quantitative method to evaluate different social groups' environmental exposure and apply the method in a case study on the COVID-19 exposure in Guangzhou, China. Severe Acute Respiratory Syndrome Coronavirus 2 (SARS-CoV-2) and its variants have ignited the COVID-19 pandemic. After infection, virus carriers have a long incubation period before onset, which causes a wider spread [18]. During this epidemic, traveling to other countries or regions usually requires mandatory quarantine according to local regulations [19]. From a policymaking or population perspective, people who come from infected areas are uniformly considered to be at risk of carrying the virus or being sick; therefore, they must be in a mandatory quarantine and tested for the virus. Therefore, in our case, we choose COVID-19-infected areas as the risk environment, and environmental exposure is defined as visits to these areas. The exposure of different social groups is then quantified by our proposed method. Our work is divided into three parts. The first is the construction of vector fields (Please see the methods in Section 3 for more details). Second, we combine the vector fields with environmental factors to model the environment exposure at a population level and propose two indicators, i.e., the total exposure indicator (TEI) and the average exposure indicator (AEI), to measure the overall exposure of various social groups. Finally, we apply this method to a COVID-19 exposure case study to explore various socioeconomic groups' exposure. The findings demonstrate the applicability of our vector-field-based approach as a visually intuitive representation to visualize an exposure map, and the results show that various groups have inequity in COVID-19 exposure.

The research significances are mainly in the three aspects: methodology, application, and social impact. Our research improves the vector field method to make it suitable for environmental exposure. Additionally, it has an improvement over other methods because it considers more elements of ALD, which is regarded as the source or mechanism that causes the spatial injustice, as noted by Soja [5]. Then, our study extends the application of vector field methods to environmental exposure. We use the evidence-based method in the case study of the early stage COVID-19 pandemic in Guangzhou to show different social groups' exposure disparities and plot the spatial distribution of exposure on a map. Our work also provides a new angle for observing the environmental exposure imbalance between various social groups, which promotes a further understanding of spatial justice and encourages a greater focus on vulnerable groups. The remainder of this paper is structured as follows. In Section 2, we review previous and related work. Section 3 presents the main methods of this study, including the method for vector-field generation and environmental-exposure modeling at the population level. In Section 4, a case study includes the study area, the data source, and the experiment process. The results are presented in Section 5. Discussion and conclusion are to be found in Section 6.

\section{Related Work}

\subsection{Spatial Justice}

In spatial justice, Soja [5] argues that the accumulation of locational decisions (ALD) is the source of inequality tilting toward a certain social group. People's various locational decisions are limited by their social roles, e.g., low-income people are more likely to go to some cheap markets to spend money, and different ALDs further cause different opportunities to come into contact with social or environmental benefits/risks, resulting in the aggravating of the inequality. 
For assessing the disparity between different social groups, the population level (or an aggregated level) is a better perspective than studying the case of a specific person, i.e., an individual level [20]. There are many previous pieces of research conducted at a population level, involving various research topics. For example, Gross et al. [21] discuss the mortality of different ethnicities from COVID-19 from a population perspective; Cwalina et al. [22] analyze the racial disparities and tobacco-related risks at a population level for risk assessment.

Spatial properties should be taken into account when studying urban problems. Due to the unbalanced resource distribution in space and various people's activity patterns, people have different opportunities to use it, which further causes inequity. For example, Jian et al. [23] pay attention to the inequity of public, open-space usage and propose a framework to assess the performance of spatial justice about public open space; Liu et al. [24] evaluate the inequity of public transportation's accessibility to medical resources and reveal the positive correlation with housing prices.

People's locational decisions involve their mobility, housing, employment, etc. [25], and it is a realistic reflection of people's plans, social situations, and deeper psychosocial reasons. For example, some researchers have investigated the psychosocial reasons why people in the slum of Dhaka are trapped and immobile [26]. Some researchers have focused on decision-making and applied it to on-demand mobility services [27].

\subsection{Environmental Exposure}

Environmental exposure problems are related to people's health, and many previous studies have tried to explore how to evaluate it, how to predict it, and what influence it has on our society [28-30]. Researchers have developed many methods to try to answer these questions; however, there is no unique method suitable for all cases. The methods should match the corresponding research questions. Our research question is whether there is a better method to describe the environmental exposures of different social groups and thus use it to explore the inequities involved. So, the quantifying method should fully consider ALD as mentioned in Section 1.

We apply our proposed method to a COVID-19 case study, and many previous works have explored the quantification of environmental exposure in pandemics. Huang and Kwan [31] used three methods to evaluate the COVID-19 exposure, including the mobilitybased approach, residence-based approach, and activity-space approach. However, these three methods, and especially the activity-space approach, are mainly targeted to individuals, which is not suitable for our population-based research question. Cartaxo et al. [32] evaluated the exposure risk through 67 political, social, economic, and natural indicators, and thus classify countries into high-risk and low-risk groups. The method takes a population perspective to study the exposure; however, there are no spatial properties, which ignores the impact of locational decisions on fairness. Kulu and Dorey [33] investigated the spatial patterns of infection by administrative area, and through spatial regression analysis, they found that urban, low-socioeconomic groups would be more affected. Elson et al. [34] argue that the administrative area statistic less reflects the variation in space, and therefore, they adopt the kernel density estimation method to realize a spatially continuous risk estimation. They utilize the population as a weight in their model to reflect the spatial heterogeneity of the influence on the population. This weighted overlay analysis approach can effectively link the environment and people affected by environmental exposures, which is also widely used in other aspects of exposure studies [29]. Our method also exploits the above-mentioned thinking to explore the environmental exposure between different social groups. Nevertheless, these two approaches focus more on the static spatial distribution and less on the dynamic interaction or mobility processes. Therefore, for filling the gaps, we introduce the vector field to better understand environmental exposure to explore our research question. 


\subsection{Vector Field}

Miller and Bridwell [15] noted that a field-based representation of continuous space could be more useful and visually intuitive and would thus provide different and valuable insights in various fields, including urban migration, urban morphology, and human mobility. As a result, they propose a vector field theory to depict the temporal-geographic urban spatial characteristics at the individual level. Liu et al. [16] extended the field theory to a population perspective, in which the vector field represents the momentum of travel demand, and therefore is also called a momentum vector field. The method is further applied by Liu et al. [17] in transportation research combined with the massive taxi global positioning system (GPS) location data from Beijing, China. A vector field fully covers space, each location has its intensities and directions, and it is also easy to calculate using vector algorithms.

Here, we briefly describe the construction of the vector field of Liu et al. [16,17]. In the process, they split trajectory-based travel vectors by small-time slot and project them on 2D space as "travel momentum", of which the modulus and direction of the vectors are determined by the method of kernel density and vector addition, and then for studying taxi congestion, the researchers project the vectors on POIs in real-time. The travel vector has two components. One is the magnitude estimated from kernel density function based on the intensity of origin and destination (OD) of trips, and the other one is the direction determined by the OD. The generation of vector field adopts the distance decay methods, which cover the whole geographical space, and the vector field is highly computational, operable, and presents the advantages of representing urban space in nature. Moreover, the method has the advantage of taking a population perspective and spatial properties into account for a better understanding of group behavior. However, it lacks consideration of the locational decisions and the trade-offs in travel when constructing vector fields, and a direct application of the original construction method may not be as applicable in environmental exposure problems. Therefore, we improve the vector field method with full consideration of ALD and make it particularly suitable for modeling environmental exposure.

\section{Methodology}

A vector field can be used to model travel intensity and direction, which can be represented by a vector's modulus and direction, respectively. In this study, we improve the vector-field-construction method for environmental exposure research, and additionally, we integrate it with decision making. Interested readers are encouraged to refer to previous related studies [15-17]. Our methodology framework is divided into three stages, as shown in Figure 1. First, we define individual vector fields. Second, we accumulate these individual vector fields to represent travel demand at the aggregated level. Third, indicators are proposed to evaluate the environmental exposure by integrating the environmental factors and the vector-field-based travel demand.

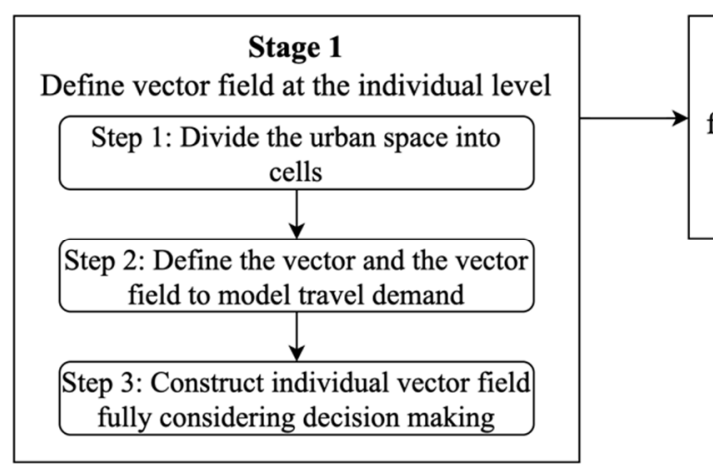

\begin{tabular}{|c|c|}
\hline $\begin{array}{c}\text { Stage 2 } \\
\text { Integrate individual vector } \\
\text { fields as a new vector field to } \\
\text { represent travel demand at } \\
\text { aggregated level }\end{array}$ & $\begin{array}{c}\text { Stage 3 } \\
\text { Propose indicators } \\
\text { for environmental exposure } \\
\text { evaluation }\end{array}$ \\
\end{tabular}

Figure 1. Methodological framework. 


\subsection{Vector Field at the Individual Level}

This section illustrates the definition of a trip vector and the individual vector field in the context of an environmental exposure study. In transportation research, Liu et al. [17] constructed a trip vector using two consecutive human activity locations. After dividing the study area into cells, these trip vectors were projected onto each cell center. Through the vector addition rule, the projected vectors in each cell can be accumulated into one vector. All vectors on the study area together form a vector field.

To suit the context of environmental exposure, we made some modifications when creating a vector field. We constructed it based on the following assumptions:

Assumption 1. The effect of environmental exposure is not a short-term process.

Assumption 2. The locations in people's fixed travel plans are regarded as familiar places, and people's movements are based around familiar places. The environmental exposure of shuttling between two familiar points is not taken into account.

Many previous environmental exposure studies also adopt non-real-time preconditions, especially from a population perspective [35-37]. So, we can also adopt a similar prerequisite, i.e., Assumption 1. Morrison et al. [38] assess the environmental exposure based on people's residence and activity locations, and there are some previous studies at the population level that do not consider intra-transportation exposure [29,34]. With the support of these previous studies, Assumption 2 can be accepted as another prerequisite. Based on these two assumptions, our approach can construct vector fields based on some relatively fixed activity points.

About the process of vector field construction, first, the study area is divided into cells. And second, we define the unit vector of which direction is from the location of human activity to the center of each cell. Then, these unit vectors are projected onto each cell center. Based on the distance between these two points, the vectors are scaled by a local distance decay function. Similarly, other human activity points can also generate corresponding vectors in this cell. Finally, we use the vector addition rule to aggregate all of an individual's co-cell vectors into one. In this way, we obtain an individual's vector field.

In the following paragraphs, we explain in detail how to define the vector fields for individuals. This process comprises the following three steps: (1) dividing the urban space into cells; (2) defining the vector and the vector field; and (3) creating the vector field for individuals.

\subsubsection{Divide the Urban Space into Cells}

Unlike the real-time application in transportation research, environmental exposure has long-term effects. Therefore, in this study, the time resolution is 1 day. In regard to space, we divide the study area into $200-\mathrm{m}$-diameter, hexagonal cells. A hexagon is suitable as the shape of spatial units for the studies involving direction [39], and $200 \mathrm{~m}$ is about the size of a residential block. For scale consistency, activity points are projected to cells, and the centroid of each cell is regarded as an activity point instead of the real point in the questionnaire. We calculate and represent the vector field based on these cells.

\subsubsection{Define the Vector and the Vector Field}

The vector field is composed of many single trip vectors. A vector has a modulus and a direction, which are determined by three elements: (1) the real or physical meaning of the vector; (2) the vector projection (including its effective range, pan, and zoom); and (3) the vector addition. In our study, a vector is a tool that shows one person's demand to travel to a certain point. The prevalent driving force is the nature of the vector field. Moreover, it is assumed that people move around based on familiar places and that they can access any public space. Therefore, the travel/trip vectors are created based on human activity locations. For clarity of representation, vectors need to be projected onto another position, i.e., each cell center. Liu et al. [17] set a given radius range and deleted the vectors outside this range when building their vector field. However, due to the wide time resolution 
in our case, we removed the given radius limitation to assign vectors to the whole study area. Different activity locations can separately generate their vectors on a place. Multiple vectors in one place are aggregated using the vector addition rule. (See Section 3.1.3 for details.) In the following paragraphs, we introduce in detail the process for defining the direction and modulus of a vector.

The direction of a vector is based on a human activity location, and it points to a cell center. Liu et al. [16] built a vector field based on the trajectories used in their transportation study. Transportation deals more with the deployment of resources in the city, and therefore the trajectory does make sense, whereas, as mentioned in Assumption 1, environmental effect on people is a long-term process. The sample trajectories only reflect people's action decisions on that day, but their route decisions may change on other days. The sample trajectories may not suit the representation of a long-term, human activity process. Assumption 2 suggests that people are active around fixed or familiar places for a long time. Therefore, we de-emphasize the role of the trajectory and place a greater emphasis on activity points. Over the long-term, people mainly carry out activities in or around familiar activity places and thus tend to travel to surrounding places starting from these familiar points. We create unit vectors that have directions from human activity locations to each cell center. We then project these vectors onto each cell center, as shown in Figure 2. Each vector radiates outward from the starting point (activity location) in the center.

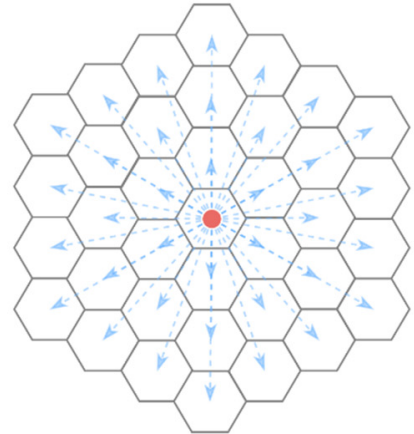

(a)

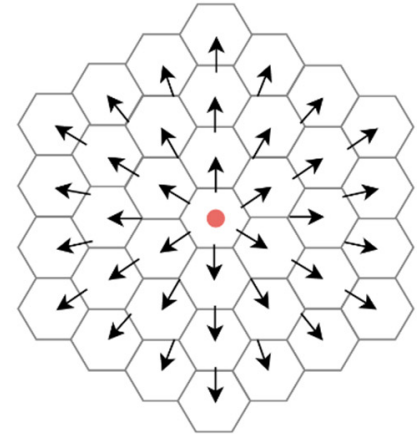

(b)

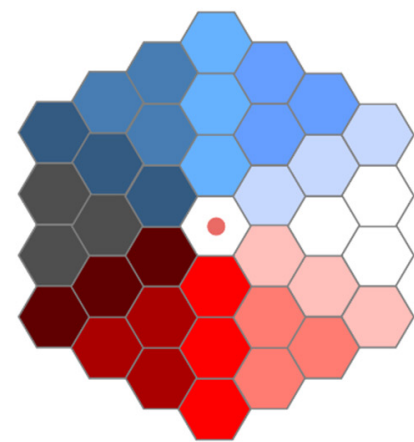

(c)

Figure 2. An activity location (red point) and the unit vectors generated based on this location. The honeycomb represents a view of the activity location from above. There are three functions for visualization: (a) The arrows represent the unit vector, but the length of each arrow is not the same, which could easily confuse readers; (b) these unit vectors are projected on the cell center; the arrows point to the centroids of destination cells, and the length of each arrow is the same; (c) the figure uses color to represent the directions, such that blue and red represent the northern and southern directions, and dark and light represent the western and eastern directions, respectively.

About their modulus, the vectors are scaled by a distance decay function, which in this case, is the probability density function (PDF) of Guangzhou's displacement distribution, rather than a simple quadratic or cubic decay function. The citizens' travel distances vary in length, and the number of trips of various distances conforms to a certain distribution, which is known as the displacement distribution. In general, as the travel distance increases, the number of people decreases. The displacement distribution better reflects human mobility patterns [40] and is suitable for the estimation of citizens' potential activity trends. Hence, the PDF of displacement distribution is used as the distance decay function in the vector field model. In a previous study, the PDF of travel distances can be fitted by a powerlaw function or by an exponential function that varies around the world [40]. Therefore, we fully exploit the distances in the participants' travel origin-destination records to obtain the PDF of the displacement distribution of Guangzhou City. The process is shown in Figure 3, and the PDF function is $P(X)=e^{-0.167 x-3.575}$, where $x$ is the displacement distance in kilometers. The process of obtaining the vector modulus involves calculating the straightline distances between the human activity locations and each cell center. Then, the scale 
factors are obtained via the distances and the function. The unit vectors are weighted by multiplying them by the scale factors, which gives the final vectors. The vectors compose the vector field as shown in Figure 4.

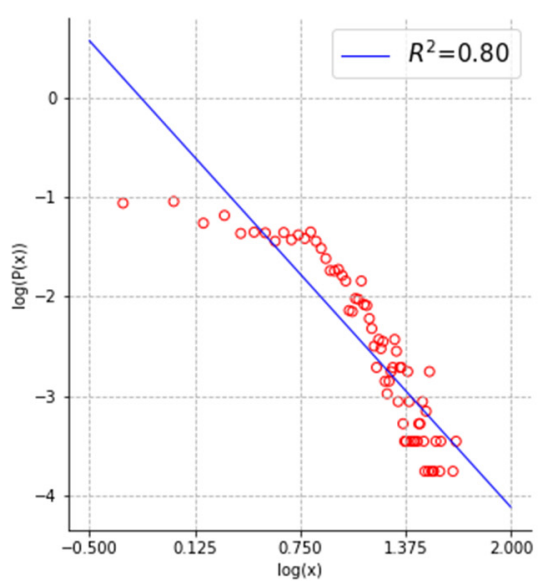

(a)

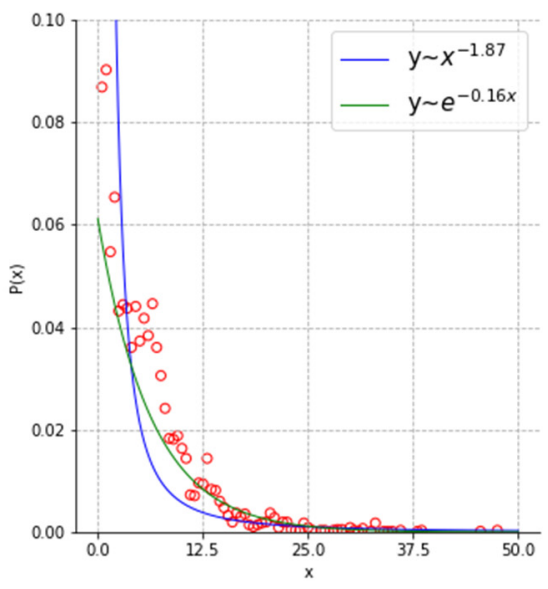

(b)

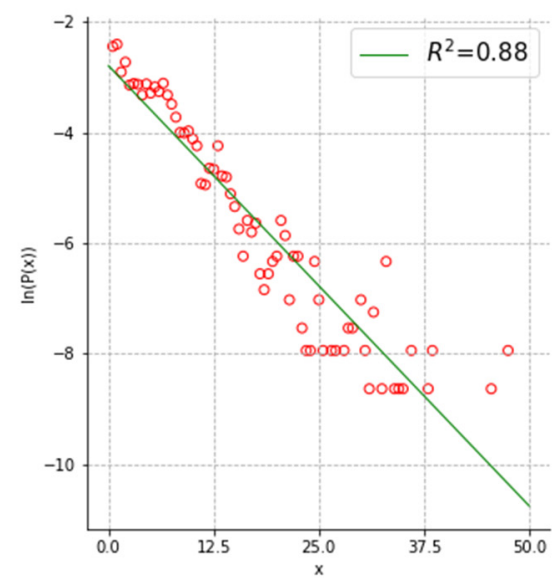

(c)

Figure 3. Displacement distribution: (a) the relationship between $\log (P)$ and $\log (x)$; (b) fitting using a power-law function and an exponential function; and (c) the relationship between $\ln (P)$ and $x$. We calculate the probability $P(x)$ of various displacements $x$ (in kilometers) at each level (from 0 to $50 \mathrm{~km}$ in increments of $0.5 \mathrm{~km}$ ) and plot the probabilities on a coordinate system (red circles). We fit the probabilities separately with a power-law function (blue line) and an exponential function (green line). After using a logarithm to transform these into linear forms, we obtain the coefficients of determination ( $R^{2}$ values) for the power-law function (0.80) and the exponential function (0.88). Therefore, in our case, we use the following exponential distribution function to represent the displacement-distribution probability density function of Guangzhou: $P(X)=e^{-0.167 x-3.575}$.

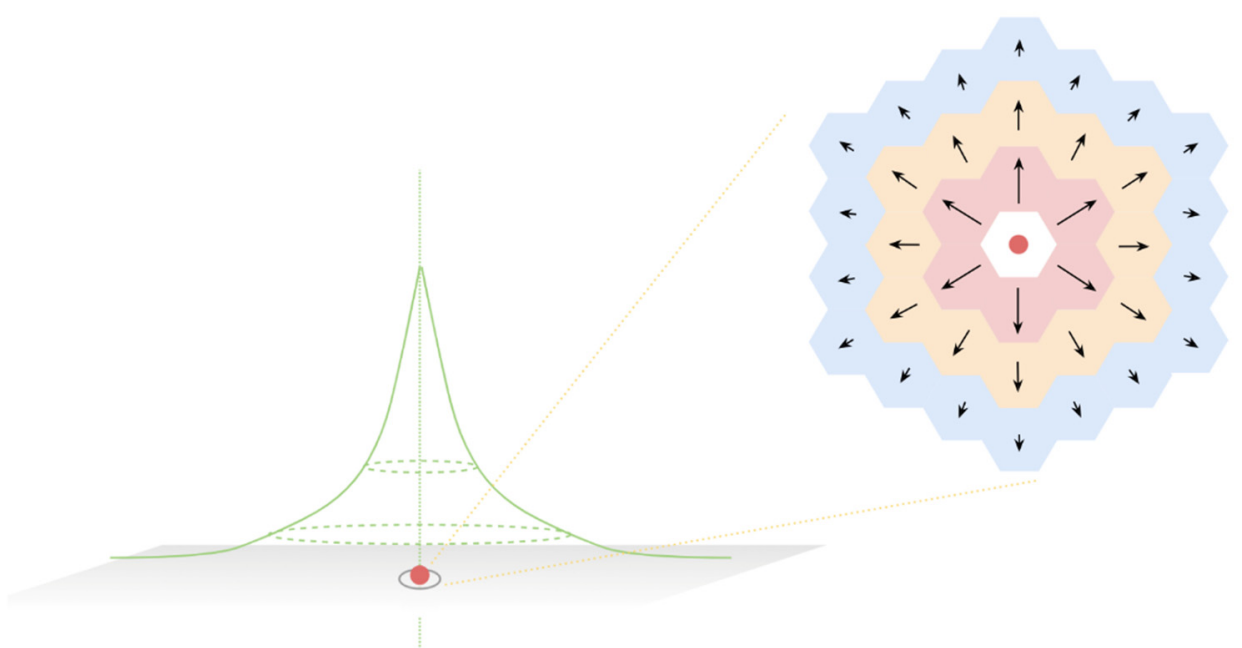

Figure 4. (Left) An activity location (red point) and its vector field. The green cone represents the vector modulus values in all positions, and these values attenuate in all directions, following the trend of the probability density function curve. (Right) Top view of the red point. The shift in color from red to blue represents the vector modulus decreasing from high to low. Arrows represent vectors, and the length and direction of each arrow show the modulus and directions of the vector it represents. The vectors are projected onto the cell center to form the vector field. 


\subsubsection{Create the Vector Field at the Individual Level}

We aggregate an individual's all activity-location-based vector fields into an individual vector field using the vector addition rule. As shown in Figure 5, the vector addition rule is used to merge two vectors to get a new vector. We argue that people's schedules or actions have decision-making costs, and people are more willing to finish their tasks at a low cost. Therefore, people tend to arrange their schedule in a reasonable way, e.g., integrating location-adjacent activities into one execution as shown in Figure 6. The example demonstrates that other activity points can affect the original schedule and travel demand. Vector addition is a suitable way to model this kind of balance or trade-off. Compared with the original vector field construction, the idea of decision-making is integrated into the vector field.

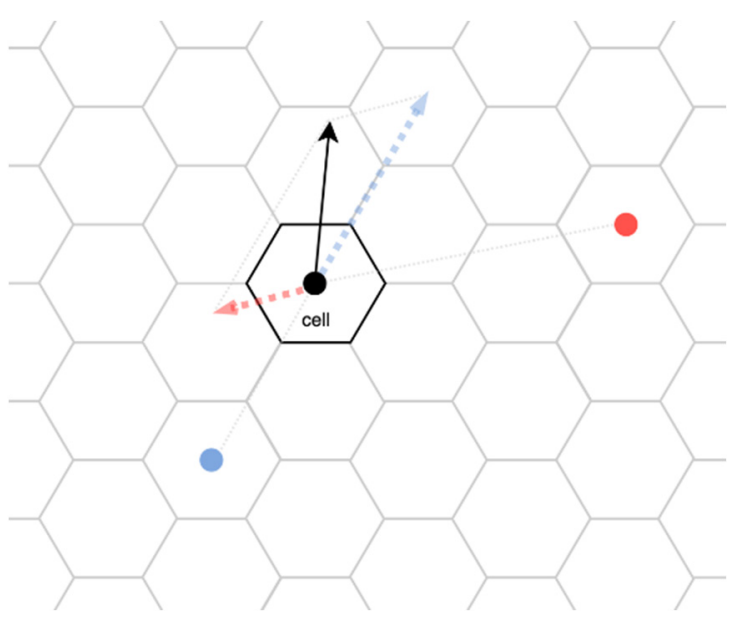

Figure 5. Accumulation of vectors for an individual. The red point generates a red vector in the black cell, and the blue point generates a blue vector in the same cell. The red and blue vectors are added using the vector addition rule, to create the black vector [41].

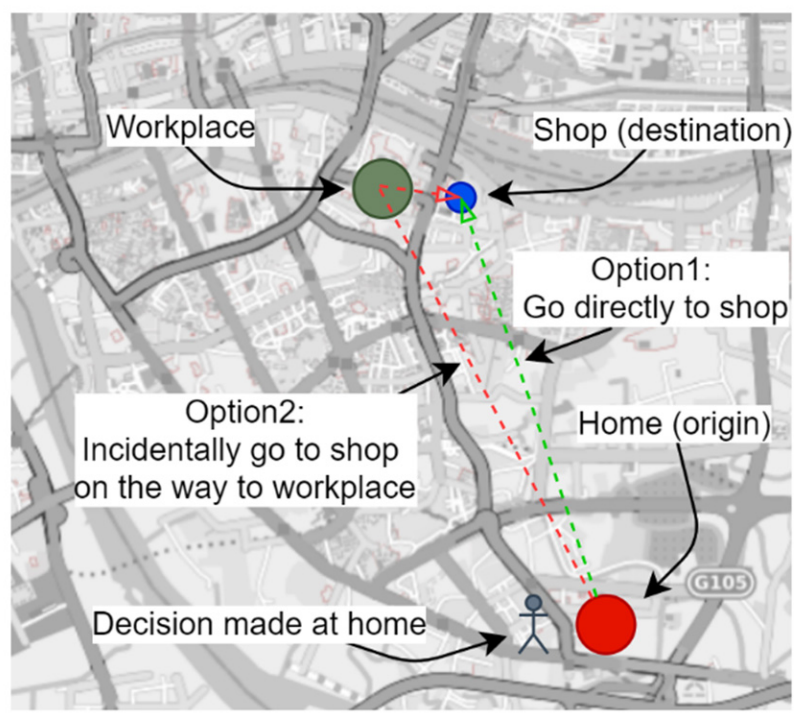

Figure 6. Here, an individual's active places are his/her home (the red point) and workplace (the green point). The shop (the blue point) is a destination on the to-do list of this individual, near his/her workplace. Because he/she will weigh or balance the cost of making a direct trip to the shop compared to incidentally going to the shop on the way to his/her workplace, the existence of his/her workplace affects his/her travel plans. 
To construct an individual vector field, we first determine all of an individual's activity points. Next, we calculate each activity point's vector field, as explained in Section 3.1.2. As multiple vectors overlap in each cell, we finally assemble these multiple vectors into a single vector with the vector addition rule to form an individual's vector field. The pseudocode in Algorithm 1 explicates the process of the vectorization and accumulation of vector fields for individuals. The input variables in the algorithm are expressed as code names: The collection of all activity points is ap_list, and the activity point ap is one of them, with the attribute of latitude ap_lat and longitude ap_lon; the collection of the centroids of cells (i.e., gridded study area) is $c$ _list, of which any a centroid point $c p$ has the attributes of latitude $c p \_l a t$, longitude $c p \_l o n$ and point's ID $i d$. The output vector field named as output contains the latitude, longitude and ID of all cells' centroids and the corresponding vectors.

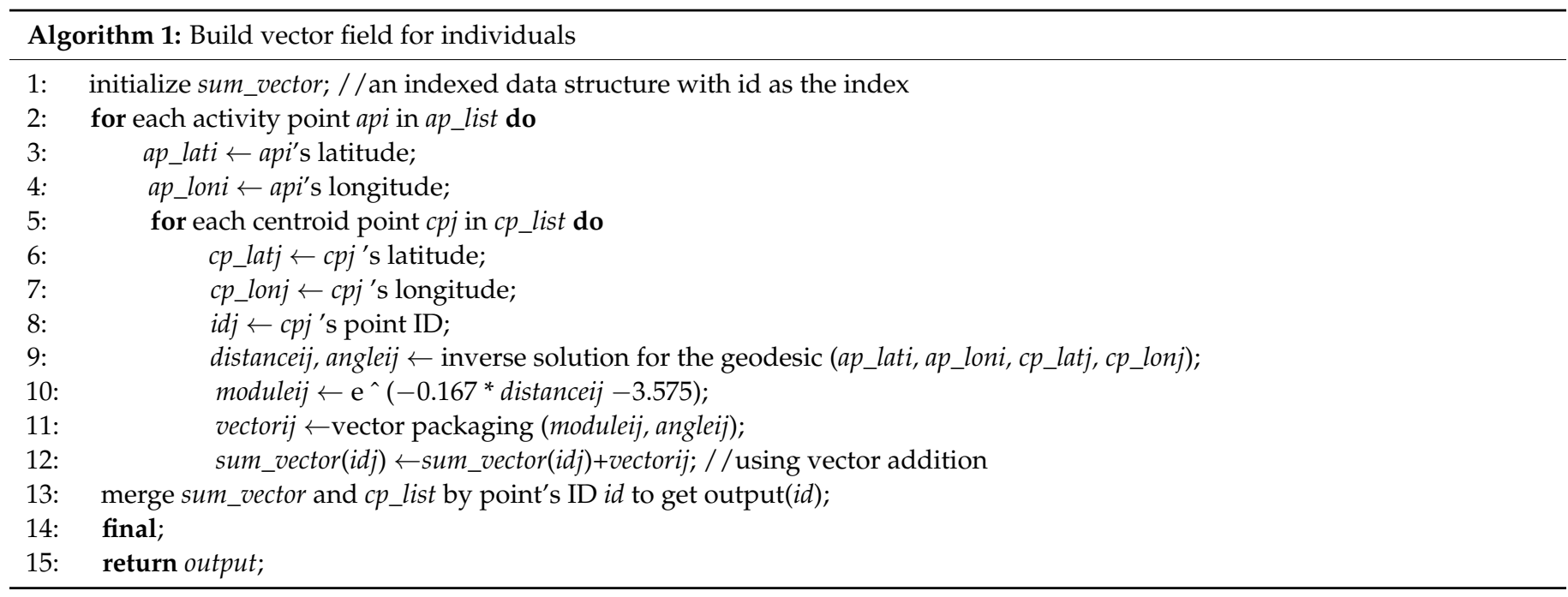

\subsection{Travel Demand at the Population Level}

In this section, we define the travel demand at an aggregated level and introduce the process of constructing it. As introduced in the above sections, we use a vector field to represent the travel demand on the individual level, and the aggregated travel demand is formed by assembling the individual travel demands. Due to the independence of people's travel decisions and schedules, an individual's travel demand is not affected by others. Therefore, it is not reasonable to use the vector addition rule to aggregate up these individual vector fields. The aggregated demand is calculated from the scalar additions of individuals' vector modulus in each cell. The pseudocode can clarify the process, as shown in Algorithm 2. The input variables in the algorithm are expressed as code names: vf_list means the collection of vector fields of all individuals (or some of them), of which individual's vector field of is an indexed data structure with the index of point's ID $i d$ and with the attributes of the vector vector. All IDs are collected in a list, id_list. vector has the attributes of module module. Output vector field output contains the latitude, longitude and ID of all cells' centroids and the corresponding scalar sum. The directions of the collective vector field are then determined with the vector addition rule. Nevertheless, it is impossible to collect all citizens' activity points. In this case, we use a questionnaire to do a sampling survey from the whole city, and the sampling result can reflect the population's (or a specific group's) character as well. 


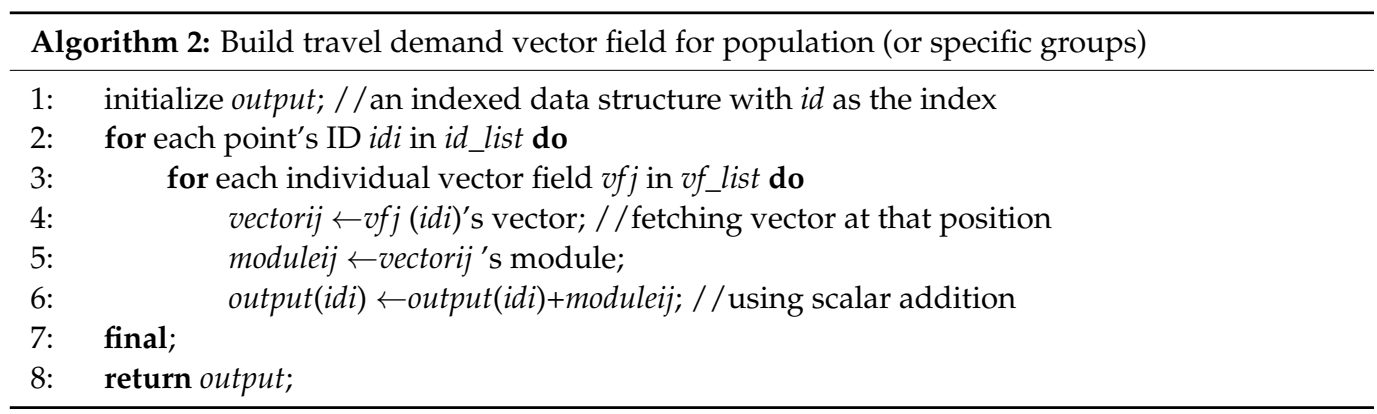

\subsection{Environmental Exposure Evaluation}

Several studies have used survey data to address the unequal effects of COVID19 on various social groups [42-44], but few are from the perspective of environmental exposure. The contextual uncertainty problem related to this was discussed in a recent health study $[45,46]$, which emphasized that an individual's spatiotemporal activity should be considered when evaluating his or her environmental exposure. We agree with this statement and further expand it to the population level. We take population or specific group's activities and environmental factors into consideration and propose indicators to evaluate environmental exposure.

We generate a new vector field by using Equation (1) in each cell. The new one integrates both travel demand and environmental factors to model the environmental exposure of a specific group (or population). To compare the degree of environmental exposure between groups, we accumulate the exposure values (i.e., the modulus of each new vector field) in all cells as a new indicator which we denote the total exposure indicator (TEI). The TEI represents the effects on the population, as shown in Equation (2):

$$
\begin{gathered}
\operatorname{modulus}_{i}=\text { Env }_{i} \cdot\left\|t d_{i}\right\| \\
\mathrm{TEI}=\frac{\sum_{i=1}^{n} \operatorname{modulus}_{i}}{n}
\end{gathered}
$$

where modulus ${ }_{i}$ is the modulus of the new vector about the exposure in position $i$; $E n v_{i}$ is the value of the environmental factor in position $i ;\left\|t d_{i}\right\|$ is the modulus of the vector about the travel demand; and $n$ is the total number of cells.

However, these evaluation criteria are greatly affected by the number of people in the various groups. To compare these groups' differences in terms of spatial distribution, we eliminate the influence of different people numbers on the results by average operation. The average vector field and the average exposure indicator (AEI) are given by Equations (3) and (4):

$$
\begin{gathered}
\text { average_modulus }_{i}=\frac{\text { modulus }_{i}}{\text { num }_{\text {people }}} \\
\mathrm{AEI}=\frac{\mathrm{TEI}}{\text { num }_{\text {people }}}
\end{gathered}
$$

where num $_{\text {people }}$ is the number of people in each group. We thereby obtain the vector field exposure map of each group and can thus analyze their similarities and differences visually. Subtraction can be conducted between maps to quantitatively (and thus more clearly) reflect the differences.

We assume that people's travel behavior does not significantly change over a long period (e.g., 2 or 3 years), and we use the densities of the communities with confirmed cases of COVID-19 as an example to demonstrate the construction of a vector-field-based exposure indicator. This model could also be extended to evaluate the other exposures by replacing the environmental factor. 


\section{Case Study}

\subsection{Study Area}

Guangzhou is among China's most developed cities. Guangzhou's inner-urban area, which has a diverse culture and population composition and the greatest density of human activity, was selected as our study area. It includes four districts-Yuexiu, Liwan, Haizhu, and Tianhe-and seventy-nine subdistricts (Figure 7).

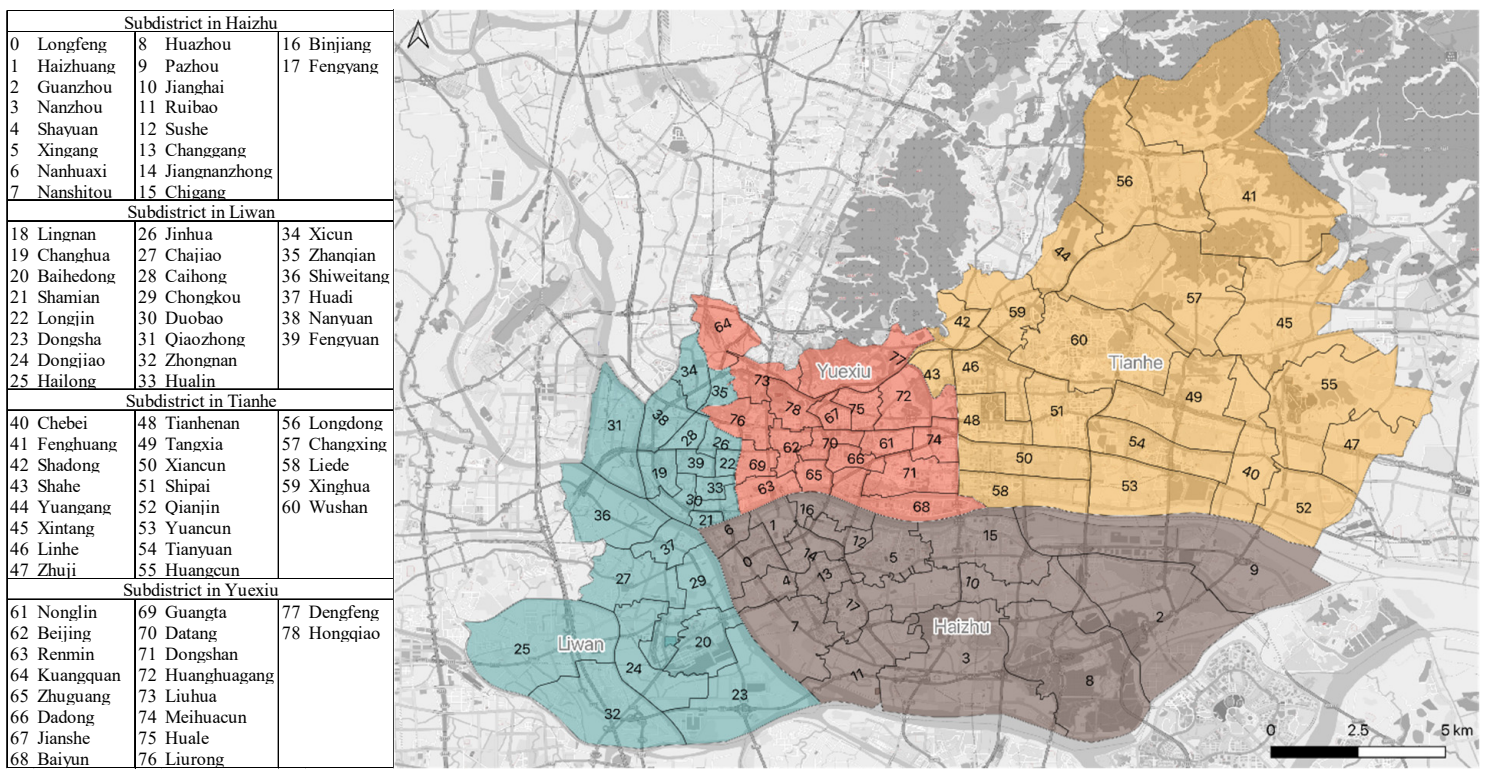

Figure 7. Study area in Guangzhou. The four districts are highlighted in different colors, the serial numbers of the seventy-nine subdistricts are labeled, and their subordination and correspondence are shown on the left.

\subsection{Dataset and Data Processing}

\subsubsection{Daily Mobility Survey}

A questionnaire survey was conducted in July 2017 to study the inhabitants' daily lives and activities. China is the first country to detect the outbreak. In the early stages of an outbreak, the crisis responses of the government and people are delayed. No one had a conception that the epidemic existed, and thus no one took any countermeasures, doing their activities as usual. According to the assumption mentioned in Section 3.1, people's habitual activities are relatively stable, so we can use the activities in the survey in 2017 to represent the activities in the pandemic non-alarm period. Participants were selected at random from the designated communities, and inhabitants younger than 18 years and older than 60 years were not included. The total number of participants was 934 . They were asked about their activities on the nearest weekday and the weekend (no more than 5 days apart). The questionnaire data include the primary key (ID), the start and end times, the latitude and longitude, and the flexibility to change the activity location (Place Change). Table 1 shows an example of the records in the survey. The question about Place Change in the survey included five levels. It assessed the participant's possibilities to change the planned activity location. A score of 1 or 2 means that the participant cannot change his/her scheduled activity location, a score of 3 means that he/she may or may not change his/her scheduled activity location (a normal situation), and a score of 4 or 5 indicate that he/she casually changes his/her scheduled activity location. In addition, some other attributes are referred to but not used in our case, including the address, partner types, activity types, and location types. 
Table 1. Examples of action records.

\begin{tabular}{cccccc}
\hline ID & Start Time & End Time & Latitude & Longitude & Place Change \\
\hline $1001-01-1$ & $3: 00: 00$ & $7: 00: 00$ & 23.127996 & 113.25522 & 1 \\
$1001-01-2$ & $7: 00: 00$ & $7: 15: 00$ & 23.127996 & 113.25522 & 3 \\
$1001-01-3$ & $7: 15: 00$ & $7: 30: 00$ & 23.127996 & 113.25522 & 4 \\
$1001-01-4$ & $7: 30: 00$ & $8: 00: 00$ & 23.127996 & 113.25522 & 3 \\
\hline
\end{tabular}

According to Assumption 1 and Assumption 2, the activity locations are expected to reflect participants' habitual activities over a long period, and we use them to construct the vector field. As shown in Table 1, the survey contains the attribute Place change, which indicates a participant's flexibility to change activity locations. We thus use the fixed activity locations on the participants' activity schedules (i.e., those with a score of 1,2 , or 3 ) to construct the vector field, comprising a total of 11,573 records.

To enable the investigation of differences in social stratification, the questionnaire includes questions on income, age, and some other social attributes. For each of these attributes, we divided the total population into two groups. For income, no clear standard is used to define poor and rich, so we use the median value (RMB 5800) to divide the population into a group with lower-middle incomes (463 people, 5963 records) and a group with higher-middle incomes ( 471 people, 5610 records). For education, the participants are ranked in the following order: (1) primary school or lower; (2) junior middle school; (3) senior middle school; (4) junior college degree: and (5) Bachelor's degree and above. The median educational attainment is a junior college degree (4), and so we define (1), (2), and (3) as the lower-middle education group (325 people, 4203 records) and (4) and (5) as the higher-middle education group (609 people, 7370 records). For age, we use the median age (35 years old) to divide the population into the lower-middle age group (463 people, 5240 records) and the higher-middle age group (470 people, 6333 records). The Migrant status is also important because local people who have lived in Guangzhou from a young age have different feelings about the urban atmosphere than do immigrants, which is also reflected by the differences in people's behavior patterns [47]. The study includes 221 immigrants with 2869 records and 713 locals with 8704 records.

\subsubsection{COVID-19 Reports}

In response to the COVID-19 pandemic, many governments disclosed the information of people infected with the virus to combat its spread. The Guangzhou Municipal Health Commission has issued a report about the COVID-19 epidemic situation every day since 22 January 2020 [48]. This report mainly contains information on the number of confirmed COVID-19 cases, the number of hospitalizations, the number of close contacts, and other information. There is a precedent for researchers to use the data extracted from articles or reports to study the spatial patterns of a pandemic's spread [49]. Before the emergence of confirmed cases, there were already some people infected by the virus in the non-alarm period. Therefore, healthy people faced a potential health risk of being exposed to the environment where the infection had appeared. As shown in Figure 8, the domestic confirmed cases increased rapidly before 24 February 2020, and the curve stabilized at 349. This shows that the pandemic was largely under control at this time. In the early community-spread stage, the names of the communities with confirmed cases were announced, but no case number was given for each community. We use the Geocode API provided by Amap [50] (accessed on 11 August 2020) to match the community's name with the location information (i.e., latitude and longitude). By conducting kernel density estimation (KDE) with a radius of $1000 \mathrm{~m}$, we generated a continuous map from 222 discrete points (i.e., communities) as shown in Figure 9. 


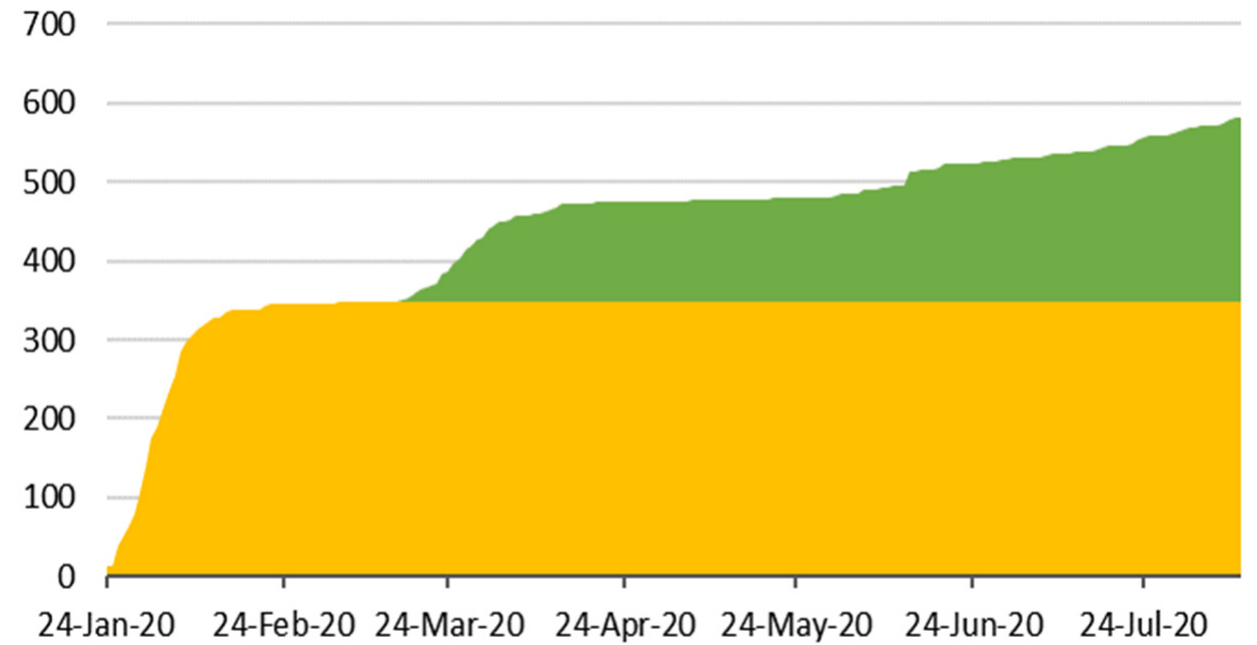

Figure 8. The cumulative curve of confirmed coronavirus 2019 (COVID-19) cases in Guangzhou, China (until 10 August 2020). The yellow curve shows the domestic confirmed cases, and the green curve shows the cases imported from overseas.

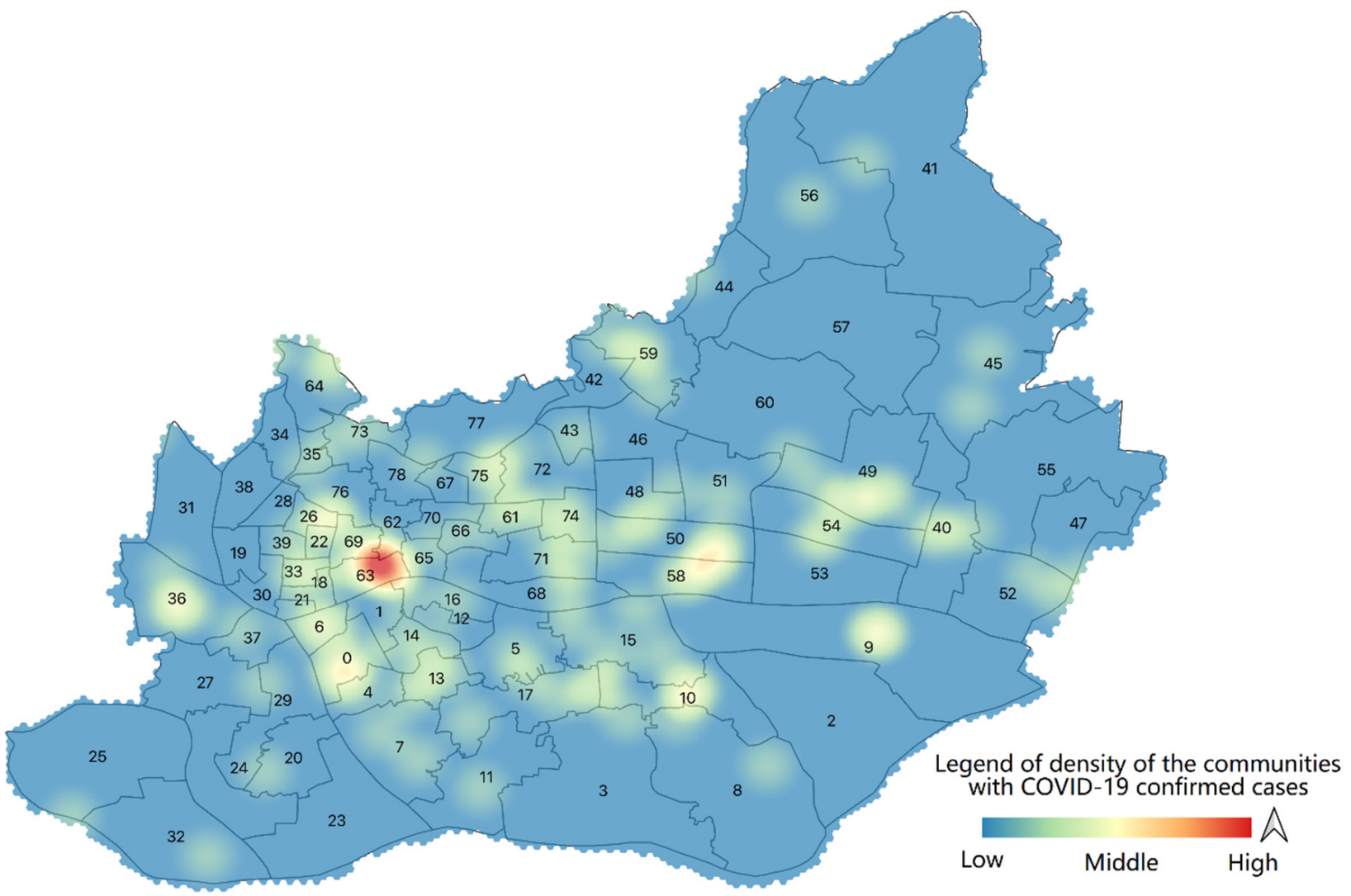

Figure 9. Interpolated density map of confirmed cases of coronavirus disease 2019 (COVID-19). 


\subsection{Constructing Vector Field to Model Travel Demand}

In this section, we introduce the process for constructing a vector field and the visualization method. Figure 10 shows the process of constructing the vector field. First, we filtered and obtained all activity locations for each group, and then gridded the entire study area with hexagonal cells via "QGIS/toolbox/Vector creation/Create grid with Grid type hexagons". Next, we judged the intersection relationship between the activity locations and these hexagonal cells, and then we substituted the coordinates of the activity locations with the coordinates of the corresponding hexagon centroids, which is realized by the function "GeoSeries.centorid" and "GeoSeries.intersection" in the Python package GeoPandas [51]. Finally, we implemented Algorithms 1 and 2 mentioned in Sections 3.1.3 and 3.2 to calculate the travel-demand vector field for each group.

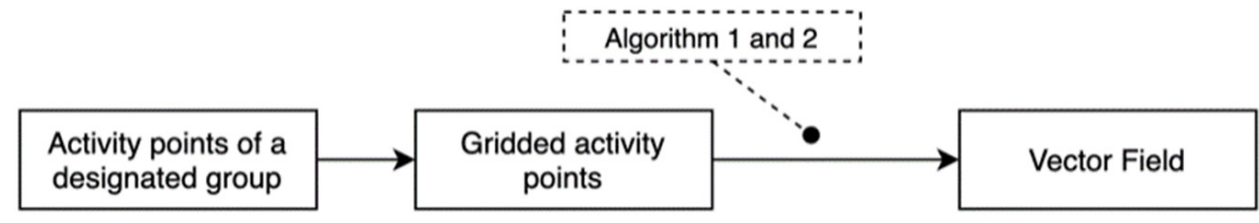

Figure 10. The process of constructing a travel-demand vector field.

A note about visualization: Although an arrow shape can naturally be used to indicate a vector, on a large-scale map, the direction and size of an arrow are illegible, and therefore we use colors instead of arrows to describe the vector field. Moreover, plotting a vector field in three dimensions (3D) can show two variables at a time, where the modulus of the vector is represented by its elevation and the direction of the vector is represented by its color. However, a 3D display would contain some distortion and occlusion, which would affect such observations. We, therefore, use contours in two dimensions (2D) to substitute for elevation in $3 \mathrm{D}$ visualization. The procedures for obtaining the raster maps and contours are as follows. We use the Inverse Distance Weighted (IDW) method [52] to generate raster maps from each group's modulus of vector fields, and we use "GDAL/raster extraction/contour" [53] in QGIS3 to generate the contour maps from these raster maps, for which the parameter "interval between contour lines" is set as 0.005 . Section 5.1 provides detailed results of our visual analysis.

\subsection{Calculating the Groups' Pandemic Exposure}

Here, we need to clarify what "environmental exposure" specifically refers to in this case. The environment here indicates a risky environment that may bring some damage to health. For example, activities in places with high air pollution can increase the risk of developing lung diseases, in which the environment refers to those areas with high air pollution, and exposure means people are active there for a long time. Similarly, according to current inbound policy in many regions, people with activities in the epidemic areas are suspected as virus carriers and need to be quarantined and tested for the virus. Therefore, in this case, the risky environment is defined as the epidemic areas and environmental exposure refers to visits to these epidemic areas. However, being exposed to a risky environment is not equal to being infected, and there is a probability between them, which can be influenced by many uncertainties. Therefore, we focus more on the assessment of environmental exposure itself and the exposure disparities of different social groups, while the amount of exposure that can lead to infection is not the concern of this paper. In Section 4.2, we have introduced a continuous map of COVID-19, which is the density of these communities with the confirmed cases. And in Section 4.3, we have constructed a vector field to show social groups' travel demands. In this section, we can combine these two parts to get a new vector field to represent the relatively high-exposure area for each group. The processing involves many spatial data operations using GIS software, and we plot a flow chart to show the whole workflow as shown in Figure 11. It mainly includes three main steps: (1) data alignment; (2) constructing the pandemic exposure 
vector field; and (3) calculating the subdistricts' AEIs and TEIs. In regard to data alignment, the environment factor map and vector field should share the same gridding system. So, we grid the environment map to get cells with KDE values. To construct pandemic exposure, Equations (1) and (3) mentioned in Section 3.3 can be used to merge the modulus of a vector field and KDE value to obtain a new value as the modulus in the pandemic exposure vector field. To calculate the subdistricts' AEIs and TEIs, we first find the cells that intersect with each subdistrict using the tool "Qgis/toolbox/vector general/join attributes by location". By using Equations (2) and (4) mentioned in Section 3.3, it is easy to calculate and obtain the values for AEI and TEI via Python. Similarly, the AEI and TEI values for the entire study area can also be calculated.

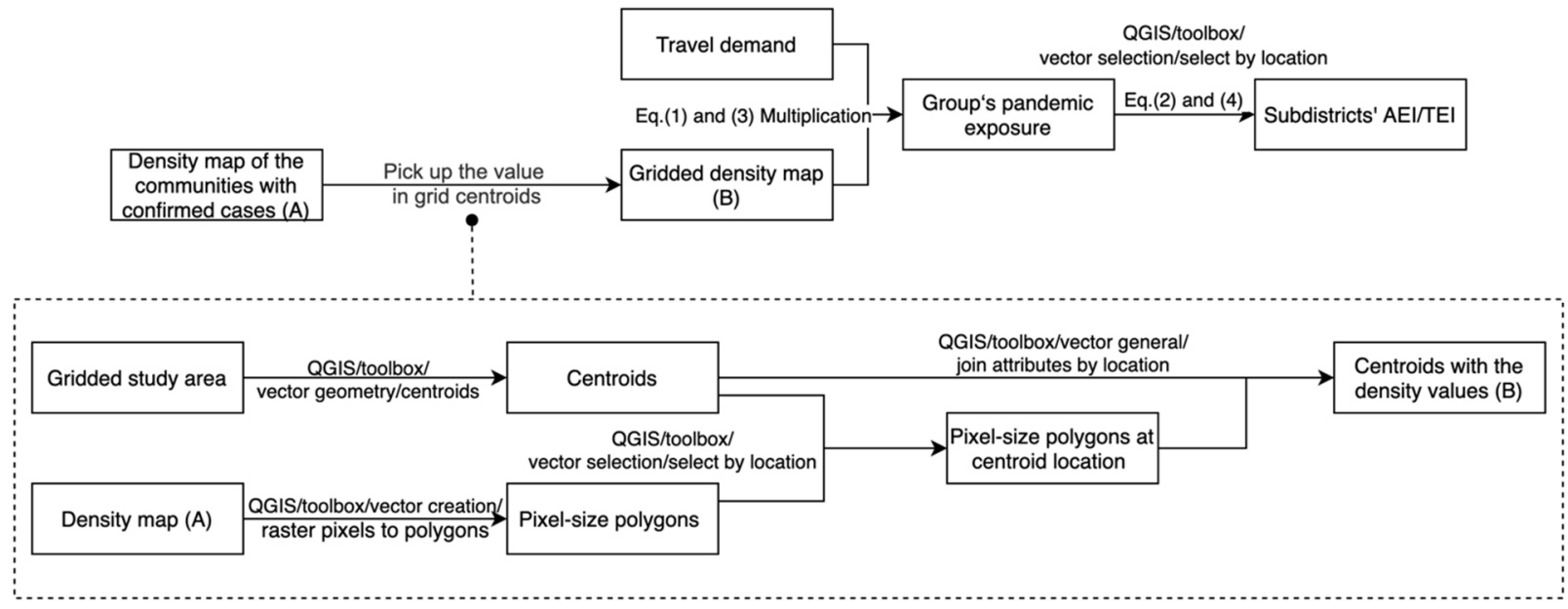

Figure 11. The workflow used to calculate the group's coronavirus 2019 (COVID-19)-exposure vector field and indicators.

\section{Result}

\subsection{Travel Demand of Each Social Group}

According to their weekday/weekend activities, we construct the vector field to model travel demand for each demographic group (Figure 12). The contour lines indicate the magnitude of the vector field (i.e., travel demand), and the colors represent the vector direction. All the maps have a similar general pattern of relatively high travel demand in the middle of the city and lower values further from the center. Multiple centers are especially prominent in weekend maps (e.g., Figure 12d). On weekdays, intra-group differences are significant. For example, the higher-middle age group (Figure 12a) displays a wider spatial coverage than the lower-middle age group (Figure 12b). In the highermiddle age group's map, there are red rings in the center of the city. The red rings mean the area has a very high value in the vector field. The lower-middle age group map does not have the red rings. A pair of the symmetric spatial distribution of high activity intensity is observed in the groups with high and low incomes and local and non-local citizens (immigrants), that is, the higher-middle income group and the local group are more active in the center area, while the lower-middle-income group and the non-local group have a similar spatial distribution mainly active in the northwest area, suggesting their different spatial behaviors and preference. On weekends, however, the intra-group differences are small. High-magnitude activities are mostly located in the city center (i.e., Zone 68, Zone 5, and Zone 15). The spatial patterns on weekends are less diverse. 


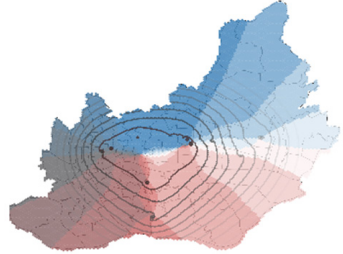

(a) weekday-high-age

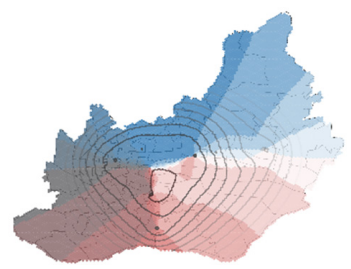

(e) weekday-high-education

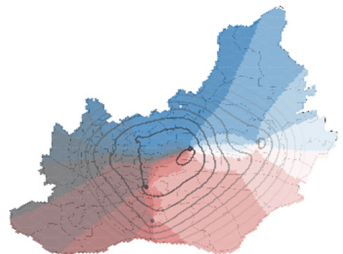

(i) weekday-high-income

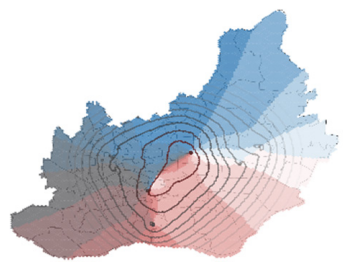

(m) weekday-immigrant

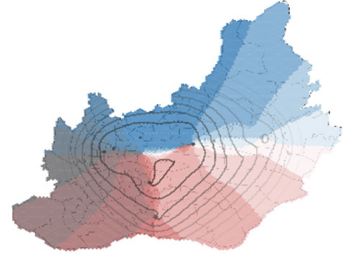

(b) weekday-low-age

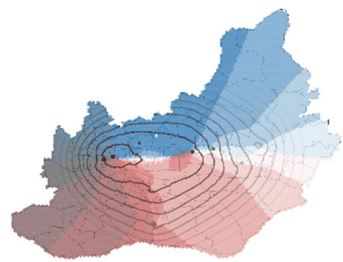

(f) weekday-low-education

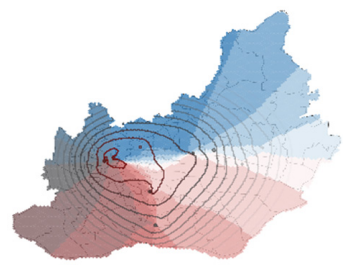

(j) weekday-low-income

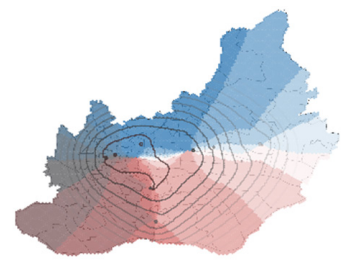

(n) weekday-locals

Legend of the contour about vector modulus

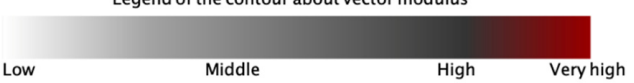

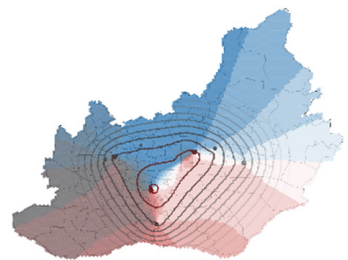

(c) weekend-high-age

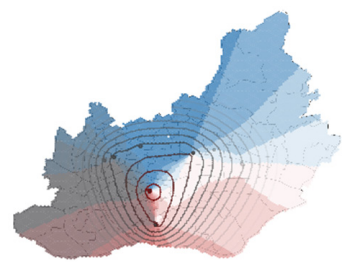

(g) weekend-high-education

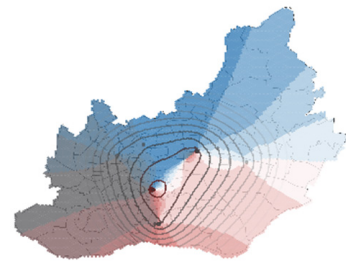

(k) weekend-high-income

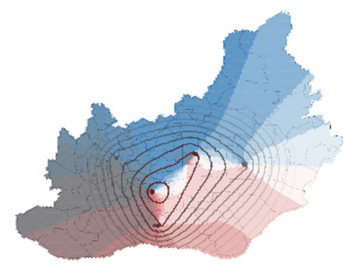

(o) weekend-immigrant

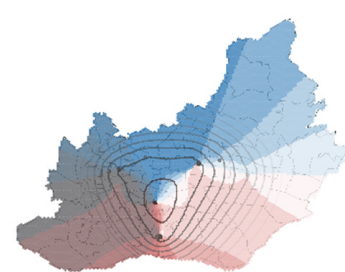

(d) weekend-low-age

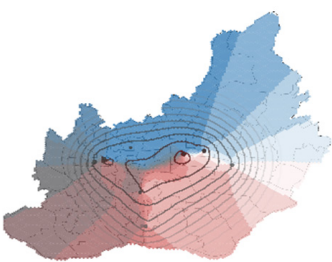

(h) weekend-low-education

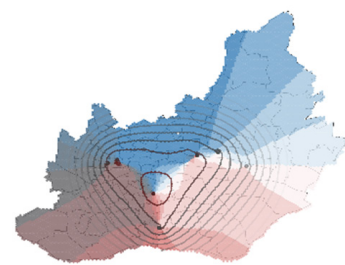

(l) weekend-low-income

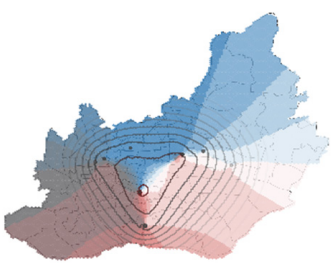

(p) weekend-locals

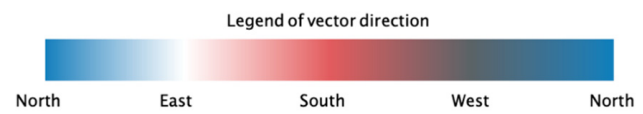

Figure 12. Travel-demand vector field for social groups on weekdays and weekends.

\subsection{Pandemic Exposure Evaluation and Comparison for Various Demographic Groups}

Using the data-processing flow described in Section 4.4, we can obtain results for AEI and TEI that reflect the pandemic exposure for all groups (Table 2). Overall, the local population on a weekday has the greatest exposure (TEI $=2.624)$, followed by those with higher education on a weekday (TEI = 2.202); and the exposure of immigrants on weekends and weekdays is the lowest (TEI $=0.646$ and 0.831 , respectively). Removing the effect of people number in each group, the low-age group on weekends and the high-income group on weekends have the lowest exposure (AEI $=3.48 \times 10^{-3}$ and $3.49 \times 10^{-3}$, respectively). In contrast, the low-income group on weekdays and the low-education group on weekdays have the greatest exposure (AEI $=3.94 \times 10^{-3}$ and $3.89 \times 10^{-3}$, respectively). In general, the exposure on weekends is lower than that on weekdays; the low-income, low-education, high-age, and immigrant groups have a greater exposure; and the high-income and low-age groups have lower exposure. In particular, compared with the high- and low-income groups on weekdays and weekends, the exposure of the low-income group on weekdays is $\sim 10 \%(9.7 \%)$ higher than that of the high-income group, and the exposure of the low-income group is $8 \%$ higher than that of the high-income group on the weekends. 
Table 2. Average exposure indicator (AEI) and total exposure indicator (TEI) of each group.

\begin{tabular}{|c|c|c|c|c|c|}
\hline Name & AEI $\left(\times 10^{-3}\right)$ & TEI & Name & $\operatorname{AEI}\left(\times 10^{-3}\right)$ & TEI \\
\hline Weekday-high-income & 3.59 & 1.67 & Weekend-high-income & 3.49 & 1.19 \\
\hline Weekday-low-income & 3.94 & 1.79 & Weekend-low-income & 3.77 & 1.35 \\
\hline Weekday-high-age & 3.81 & 1.77 & Weekend-high-age & 3.78 & 1.35 \\
\hline Weekday-low-age & 3.71 & 1.68 & Weekend-low-age & 3.48 & 1.19 \\
\hline Weekday-high-education & 3.69 & 2.20 & Weekend-high-education & 3.61 & 1.60 \\
\hline Weekday-low-education & 3.89 & 1.25 & Weekend-low-education & 3.68 & 0.94 \\
\hline Weekday-migrant & 3.83 & 0.83 & Weekend-migrant & 3.71 & 0.65 \\
\hline Weekday-local & 3.74 & 2.62 & Weekend-local & 3.61 & 1.89 \\
\hline
\end{tabular}

\subsection{Pandemic Exposure Evaluation and Comparison in Space}

For ease of demonstration, we have selected income groups as an example. After calculating the subdistricts' AEI and TEI for four groups, we can conclude the areas with the highest and lowest pandemic exposures. Coincidentally, as shown in Table 3, the top three and bottom three are consistent in four groups: Renmin (Zone 63) has the highest exposure, followed by Guangta (Zone 69) and Longjin (Zone 22); and Changxing (Zone 57) has the lowest exposure, followed by Dongsha (Zone 23) and Guanzhou (Zone 2). These high-exposure areas (i.e., Zones 22, 69, and 63) are close to each other in the center of the city. As shown in Figure 13, we illustrated the vector fields of various income groups on the weekday or weekend.

Table 3. The subdistricts with the highest and lowest pandemic exposure.

\begin{tabular}{|c|c|c|c|c|c|c|c|c|c|c|}
\hline \multirow{2}{*}{ Rank } & \multirow{2}{*}{ Name } & \multirow{2}{*}{ ID } & \multicolumn{2}{|c|}{ Weekday-High-Income } & \multicolumn{2}{|c|}{ Weekday-Low-Income } & \multicolumn{2}{|c|}{ Weekend-High-Income } & \multicolumn{2}{|c|}{ Weekend-Low-Income } \\
\hline & & & AEI $\left(\times 10^{-3}\right)$ & TEI & AEI $\left(\times 10^{-3}\right)$ & TEI & AEI $\left(\times 10^{-3}\right)$ & TEI & AEI $\left(\times 10^{-3}\right)$ & TEI \\
\hline 1 & Renmin & 63 & 36.97 & 17.23 & 46.24 & 20.95 & 37.23 & 12.73 & 43.27 & 15.45 \\
\hline 2 & Guangta & 69 & 27.28 & 12.71 & 34.69 & 15.72 & 27.01 & 9.24 & 32.25 & 11.51 \\
\hline 3 & Longjin & 22 & 19.64 & 9.15 & 25.06 & 11.35 & 18.34 & 6.27 & 22.56 & 8.05 \\
\hline$\ldots$ & $\ldots$ & $\ldots$ & $\ldots$ & $\ldots$ & $\ldots$ & $\ldots$ & $\ldots$ & $\ldots$ & $\ldots$ & $\ldots$ \\
\hline 77 & Guanzhou & 2 & 0.05 & 0.02 & 0.05 & 0.02 & 0.05 & 0.02 & 0.05 & 0.02 \\
\hline 78 & Dongsha & 23 & 0.01 & 0.00 & 0.01 & 0.01 & 0.01 & 0.003 & 0.01 & 0.004 \\
\hline 79 & Changxing & 57 & 0.00 & 0.00 & 0.00 & 0.00 & 0.00 & 0.00 & 0.00 & 0.00 \\
\hline
\end{tabular}

Note that AEI means average exposure indicator and TEI means total exposure indicator.

The detailed differences in pandemic exposure distribution cannot be easily visualized from individual maps. In Figure 14, we subtract the cell value of the lower-middle-income group from that of the higher-middle-income group. The green areas indicate higher exposure for the low-income group, and the brown areas indicate higher exposure for the high-income group. We find that on the weekday, low-income citizens have an overall greater exposure in the western and central zones, while the high-income group has a greater exposure in distant areas, and on the weekend, the high-income group has no higher exposure areas. 

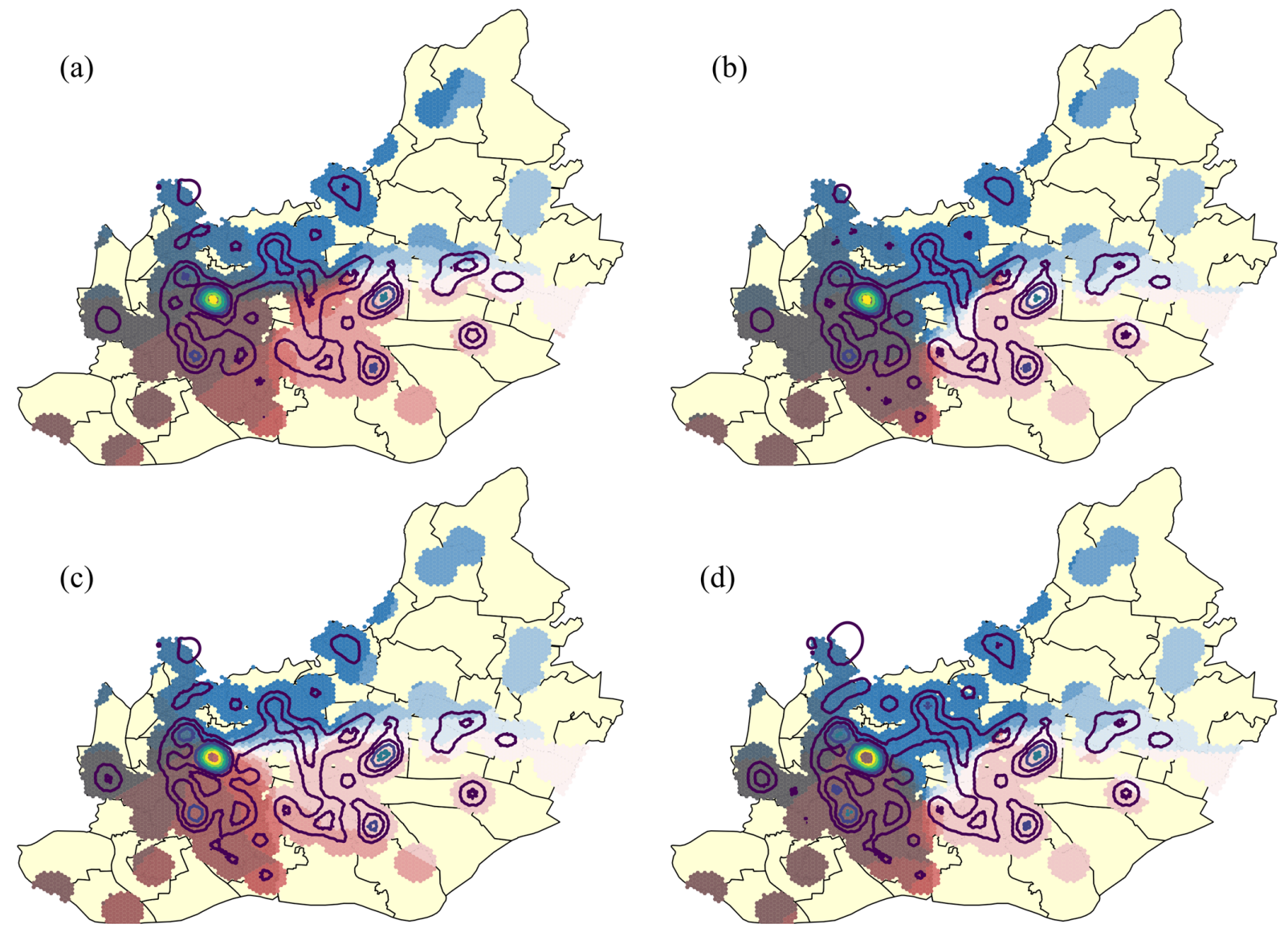

W
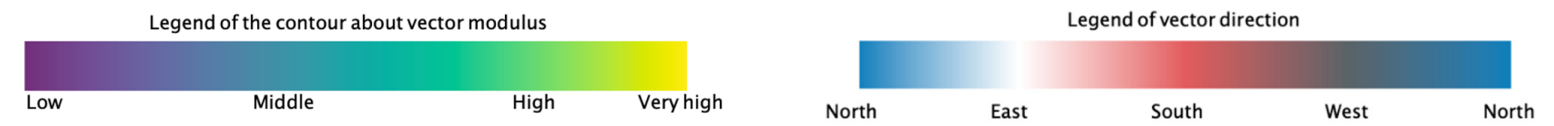

Figure 13. High- and low-income groups' pandemic exposure on weekdays and weekends. (a) higher-middle income group on the weekday; (b) higher-middle income group on the weekend; (c) lower-middle-income group on the weekday; (d) lower-middle-income group on the weekend.

(a)

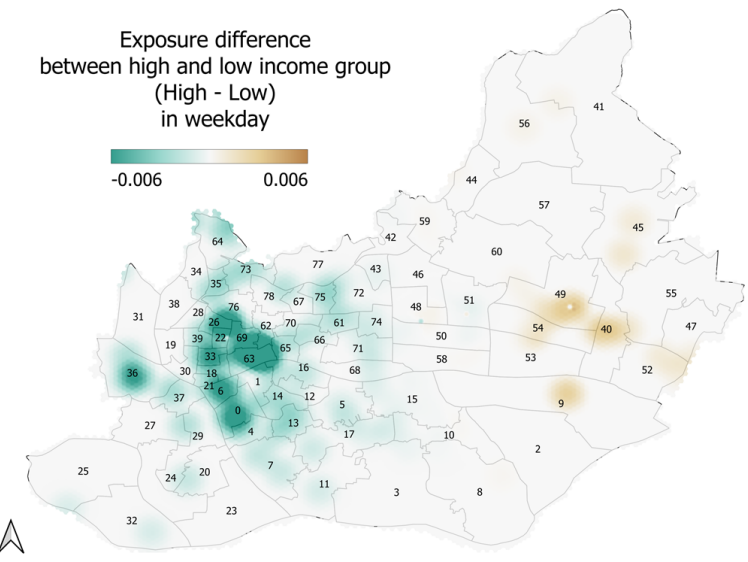

(b)

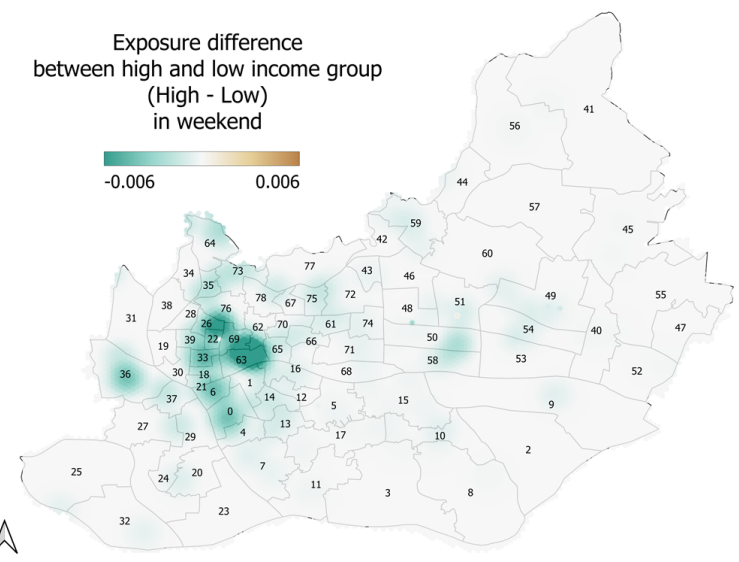

Figure 14. Exposure comparison between high- and low-income groups: (a) Weekday; (b) Weekend. 


\section{Discussion and Conclusions}

Environmental exposure is always a threat to people's health and lives, and it is necessary to quantify it. Many researchers evaluate it based on an individual perspective (Yang et al., 2020; Huang and Kwan, 2021). Nevertheless, when facing environmental exposure, inequality exists in the different levels of risk (or benefits) faced by different social groups. As such, quantification needs a population perspective. Soja argues social groups ${ }^{\prime}$ accumulation of locational decisions (ALD) determines social inequality [5]. However, the current population-based exposure quantification method considers few properties about mobility and decisions. Therefore, a vector field-based method is proposed to quantify the environmental exposure from a population perspective, covering three elements: population perspective, mobility, and decisions, and fully reflecting the thinking of ALD in spatial justice. We apply it to a case study of COVID-19 exposure and explore the inequities involved. The findings of the case study, the contribution of our proposed method, and the limitations are discussed in the following:

From the case study of COVID-19 exposure, the results indicate that various social groups differ in their pandemic exposure. The low-income, low-education, high-age, and immigrant groups have greater exposure, and the high-income and low-age groups have lower exposure. In particular, the exposure of the low-income group is $8 \sim 10 \%(9.7 \%)$ higher than that of the high-income group. From a spatial aspect, the low-income group has higher exposure in the west of the city while the high-income group has higher exposure in the east of the city on the weekday, and on the weekend, the low-income group has higher exposure in the west of the city, but none of the regions had higher exposure for the higher income groups. We find that those groups with higher exposure all belong to vulnerable groups in society. People in low-income, low-education, and immigrant groups are probably in occupations that require frequent mobility, further increasing their exposure to the epidemic. As governments restrict travel to control epidemics, their livelihoods are affected more than others, increasing further hardship and inequality. Similar conclusions are also referred to by other studies [54]. People belonging to high-income, high-education, and local groups, may have fixed-place work and their job may not force them to move around, which protects them from exposure. Compared with those in the low-age group, high-age people may have more flexible time or opportunities to travel to other places, objectively creating an increased exposure. The travels of young people may be controlled by their work and the group may not have time and energy for extra traveling, causing less contact with environmental exposure. From the comparison of the case on the weekday and weekend, we could infer that, in the workday, both high- and low-income groups may need to go out to work, resulting in higher exposure for high-income groups in some places and higher exposure for low-income groups in others. On the weekend, the higher-income groups might take a break on a day off, but the lower-income groups might still need to work, resulting in generally higher exposure than higher-income groups.

With our proposed method, we have improved on the vector-field-construction method to make it more useful to environmental exposure studies [16]. It simulates exposure; nevertheless, it is more suitable for population-based studies rather than those on an individual level. This method is an improvement compared to other methods. It has the advantage of being easy to calculate and a visually intuitive in representation, and in addition, the method attempts to integrate more key elements of ALD thinking into the quantification of environmental exposure, which is associated with spatial justice. Based on the vector field, we propose two indicators, i.e., TEI and AEI, to evaluate each group's environmental exposure. Our method provides a better understanding of the environmental exposure at a population level, revealing the disparity between various social groups in spatial distribution dimension and quantification dimension. Although we choose COVID-19 exposure as a case study, there are also diverse possibilities that other kinds of environmental exposure can be integrated with the travel-demand-vector field, not only pandemic exposure. The results can provide a useful reference for the government to select a customized and flexible control strategy. This work could serve as a 
guide for the prevention or control of pandemics during the early stages of an outbreak or during multi-wave stages. With the rapid development of information and community technologies, mobile phones and applications collect users' location information to understand their mobility. However, these data are controlled by telecom operators and software developers. Due to privacy, some data cannot be made public, and some data require high-cost purchases. It is negative for academic communication. In our case, our vector-field approach also works for small data.

The limitations of our work mainly involve four aspects. First, more fine-grained environmental factors can improve the accuracy of results. In our case study, COVID-19 exposure data are based on the community of confirmed patients. The data may ignore the other possible places with the pandemic, which may cause a certain underestimation about the exposure. If we have detailed epidemiological investigation data, the results may be able to respond better to the reality of the situation. Second, our results are derived under some assumptions, even if from a population perspective, there are still many factors not taken into account, e.g., the real-world urban context. Ignoring it will cause the uncertain geographic context problem (UGCoP) [45]. Third, in regard to the modifiable areal unit problem (MAUP), we divide the study area into small cells and construct the vector field based on these small cells. However, if one cell covers many activity points, they are aggregated into one point when constructing the vector field, which may cause inadequate data and some errors. So, if we choose a large cell size, the method has a MAUP problem. Nevertheless, if we choose a small cell size, MAUP may have a limited influence. Most of the values may not have major variations through being adjusted by the vector calculation and our proposed indicators, i.e., TEI and AEI, have been averaged by the cell numbers. In our case, the cell size is $200 \mathrm{~m}$, which is acceptable compared to the spacing of the activity points. We believe that future research may improve the analysis framework by considering UGCoP and MAUP and be applied to some other fields. Fourth, our proposed method can only assess exposure and cannot predict disease incidence, e.g., COVID-19 infection. Because many uncertainties are involved in whether or not someone exposed to the environment may get the disease, our research does not further discuss how much exposure can lead to infection, but rather we treat exposure as a relative metric to compare disparities between different social groups. An exposure-based pandemic prediction may be one of our future works.

Author Contributions: Conceptualization, Zijian Guo, Xintao Liu, and Pengxiang Zhao; Methodology, Zijian Guo and Xintao Liu; Validation, Zijian Guo, Xintao Liu, and Pengxiang Zhao; Formal analysis, Zijian Guo; Investigation, Zijian Guo; Resources, Xintao Liu and Pengxiang Zhao; Data curation, Zijian Guo; Writing—original draft, Zijian Guo; Writing—review \& editing, Zijian Guo, Xintao Liu, Pengxiang Zhao; Visualization, Zijian Guo; Supervision, Xintao Liu; Project administration, Xintao Liu. All authors have read and agreed to the published version of the manuscript.

Funding: The work was jointly supported two research projects: RGC Early Career Scheme (P0030875) and RISUD PolyU (P0038289).

Data Availability Statement: Not available.

Conflicts of Interest: The authors declare no conflict of interest.

\section{References}

1. Braun-Fahrländer, C.; Riedler, J.; Herz, U.; Eder, W.; Waser, M.; Grize, L.; Maisch, S.; Carr, D.; Gerlach, F.; Bufe, A.; et al. Environmental exposure to endotoxin and its relation to asthma in school-age children. N. Engl. J. Med. 2002, 347, 869-877. [CrossRef] [PubMed]

2. Satarug, S.; Garrett, S.H.; Sens, M.A.; Sens, D.A. Cadmium, environmental exposure, and health outcomes. Environ. Health Perspect. 2010, 118, 182-190. [CrossRef]

3. Templeton, A.; Guven, S.T.; Hoerst, C.; Vestergren, S.; Davidson, L.; Ballentyne, S.; Madsen, H.; Choudhury, S. Inequalities and identity processes in crises: Recommendations for facilitating safe response to the COVID-19 pandemic. Br. J. Soc. Psychol. 2020, 59, 674-685. [CrossRef] [PubMed] 
4. Redondo-Sama, G.; Matulic, V.; Munté-Pascual, A.; de Vicente, I. Social work during the COVID-19 crisis: Responding to urgent social needs. Sustainability 2020, 12, 8595. [CrossRef]

5. Soja, E. The city and spatial justice. Spat. Justice 2009, 1, 1-5.

6. Wiesner, M.R.; Lowry, G.V.; Jones, K.L.; Hochella, M.F., Jr.; Di Giulio, R.T.; Casman, E.; Bernhardt, E.S. Decreasing uncertainties in assessing environmental exposure, risk, and ecological implications of nanomaterials. Environ. Sci. Technol. 2009, 43, 6458-6462. [CrossRef]

7. Zhang, L.; Zhou, S.; Kwan, M.P.; Chen, F.; Lin, R. Impacts of individual daily greenspace exposure on health based on individual activity space and structural equation modeling. Int. J. Environ. Res. Public Health 2018, 15, 2323. [CrossRef]

8. Kwan, M.-P.; Wang, J.; Tyburski, M.; Epstein, D.H.; Kowalczyk, W.J.; Preston, K.L. Uncertainties in the geographic context of health behaviors: A study of substance users' exposure to psychosocial stress using GPS data. Int. J. Geogr. Inf. Sci. 2019, 33, 1176-1195. [CrossRef]

9. Coccia, M. An index to quantify environmental risk of exposure to future epidemics of the COVID-19 and similar viral agents: Theory and practice. Environ. Res. 2020, 191, 110155. [CrossRef]

10. Rodes, C.E.; Kamens, R.M.; Wiener, R.W. The significance and characteristics of the personal activity cloud on exposure assessment measurements for indoor contaminants. Indoor Air 1991, 1, 123-145. [CrossRef]

11. Su, J.G.; Morello-Frosch, R.; Jesdale, B.M.; Kyle, A.D.; Shamasunder, B.; Jerrett, M. An index for assessing demographic inequalities in cumulative environmental hazards with application to Los Angeles, California. Environ. Sci. Technol. 2009, 43, 7626-7634. [CrossRef] [PubMed]

12. Padilla, C.M.; Kihal-Talantikite, W.; Vieira, V.M.; Rossello, P.; Le Nir, G.; Zmirou-Navier, D.; Deguen, S. Air quality and social deprivation in four French metropolitan areas-A localized spatio-temporal environmental inequality analysis. Environ. Res. 2014, 134, 315-324. [CrossRef] [PubMed]

13. Milojevic, A.; Niedzwiedz, C.L.; Pearce, J.; Milner, J.; MacKenzie, I.A.; Doherty, R.M.; Wilkinson, P. Socioeconomic and urban-rural differentials in exposure to air pollution and mortality burden in England. Environ. Health 2017, 16, 104. [CrossRef]

14. Yang, N.; Fu, R.; Chao, Y.; Liu, H.; Ma, X. Quantitative assessment of environmental exposure of delivery men in Wuhan. Arch Environ. Occup. Health 2020, 75, 445-463. [CrossRef] [PubMed]

15. Miller, H.J.; Bridwell, S.A. A field-based theory for time geography. Ann. Assoc. Am. Geogr. 2009, 99, 49-75. [CrossRef]

16. Liu, X.; Yan, W.Y.; Chow, J.Y.J. Time-geographic relationships between vector fields of activity patterns and transport systems. J. Transp. Geogr. 2015, 42, 22-33. [CrossRef]

17. Liu, X.; Chow JY, J.; Li, S. Online monitoring of local taxi travel momentum and congestion effects using projections of taxi GPS-based vector fields. J. Geogr. Syst. 2018, 20, 253-274. [CrossRef]

18. McAloon, C.; Collins, Á.; Hunt, K.; Barber, A.; Byrne, A.W.; Butler, F.; Casey, M.; Griffin, J.; Lane, E.; McEvoy, D.; et al. Incubation period of COVID-19: A rapid systematic review and meta-analysis of observational research. BMJ Open 2020, 10, e039652. [CrossRef]

19. Tang, B.; Xia, F.; Tang, S.; Bragazzi, N.L.; Li, Q.; Sun, X.; Liang, J.; Xiao, Y.; Wu, J. The effectiveness of quarantine and isolation determine the trend of the COVID-19 epidemics in the final phase of the current outbreak in China. Int. J. Infect. Dis. 2020, 95, 288-293. [CrossRef]

20. Goldthorpe, J.H. Sociology as a Population Science; Cambridge University Press: Cambridge, UK, 2016.

21. Gross, C.P.; Essien, U.R.; Pasha, S.; Gross, J.R.; Wang, S.Y.; Nunez-Smith, M. Racial and ethnic disparities in population-level Covid-19 mortality. J. Gen. Intern. Med. 2020, 35, 3097-3099. [CrossRef]

22. Cwalina, S.N.; Ihenacho, U.; Barker, J.; Smiley, S.L.; Pentz, M.A.; Wipfli, H. Advancing racial equity and social justice for Black communities in US tobacco control policy. Tob. Control 2021. [CrossRef] [PubMed]

23. Jian, I.Y.; Luo, J.; Chan, E.H. Spatial justice in public open space planning: Accessibility and inclusivity. Habitat Int. 2020, 97, 102122. [CrossRef]

24. Liu, X.; Lin, Z.; Huang, J.; Gao, H.; Shi, W. Evaluating the Inequality of Medical Service Accessibility Using Smart Card Data. Int. J. Environ. Res. Public Health 2021, 18, 2711. [CrossRef] [PubMed]

25. Chan, C.S.; Shek, K.F. Are Guangdong-Hong Kong-Macao Bay area cities attractive to university students in Hong Kong? Leading the potential human capital from image perception to locational decisions. J. Place Manag. Dev. 2021, 14, 404-429. [CrossRef]

26. Ayeb-Karlsson, S.; Kniveton, D.; Cannon, T. Trapped in the prison of the mind: Notions of climate-induced (im) mobility decision-making and wellbeing from an urban informal settlement in Bangladesh. Palgrave Commun. 2020, 6, 62. [CrossRef]

27. Xie, Y.; Danaf, M.; Azevedo, C.L.; Akkinepally, A.P.; Atasoy, B.; Jeong, K.; Seshadri, R.; Ben-Akiva, M. Behavioral modeling of on-demand mobility services: General framework and application to sustainable travel incentives. Transportation 2019, 46, 2017-2039. [CrossRef]

28. Oldenkamp, R.; Hoeks, S.; Čengić, M.; Barbarossa, V.; Burns, E.E.; Boxall, A.B.; Ragas, A.M. A high-resolution spatial model to predict exposure to pharmaceuticals in European surface waters: EPiE. Environ. Sci. Technol. 2018, 52, 12494-12503. [CrossRef]

29. Xu, Y.; Jiang, S.; Li, R.; Zhang, J.; Zhao, J.; Abbar, S.; González, M.C. Unraveling environmental justice in ambient PM2.5 exposure in Beijing: A big data approach. Comput. Environ. Urban Syst. 2019, 75, 12-21. [CrossRef]

30. Song, Y.; Chen, B.; Kwan, M.P. How does urban expansion impact people's exposure to green environments? A comparative study of 290 Chinese cities. J. Clean. Prod. 2020, 246, 119018. [CrossRef] 
31. Huang, J.; Kwan, M.P. Uncertainties in the assessment of COVID-19 risk: A Study of people's exposure to high-risk environments using individual-level activity data. Ann. Am. Assoc. Geogr. 2021, 1-20. [CrossRef]

32. Cartaxo, A.N.S.; Barbosa, F.I.C.; de Souza Bermejo, P.H.; Moreira, M.F.; Prata, D.N. The exposure risk to COVID-19 in most affected countries: A vulnerability assessment model. PLoS ONE 2021, 16, e0248075. [CrossRef] [PubMed]

33. Kulu, H.; Dorey, P.S. Infection rates from Covid-19 in Great Britain by geographical units: A model-based estimation from mortality data. Health Place 2021, 67, 102460. [CrossRef] [PubMed]

34. Elson, R.; Davies, T.M.; Lake, I.R.; Vivancos, R.; Blomquist, P.B.; Charlett, A.; Dabrera, G. The spatio-temporal distribution of COVID-19 infection in England between January and June 2020. Epidemiol. Infect. 2021, 149, 1-19. [CrossRef] [PubMed]

35. Selander, J.; Nilsson, M.E.; Bluhm, G.; Rosenlund, M.; Lindqvist, M.; Nise, G.; Pershagen, G. Long-term exposure to road traffic noise and myocardial infarction. Epidemiology 2009, 20, 272-279. [CrossRef] [PubMed]

36. Wang, M.; Aaron, C.P.; Madrigano, J.; Hoffman, E.A.; Angelini, E.; Yang, J.; Laine, A.; Vetterli, T.M.; Kinney, P.L.; Sampson, P.D.; et al. Association between long-term exposure to ambient air pollution and change in quantitatively assessed emphysema and lung function. JAMA 2019, 322, 546-556. [CrossRef]

37. Larsen, K.; Black, P.; Rydz, E.; Nicol, A.M.; Peters, C.E. Using geographic information systems to estimate potential pesticide exposure at the population level in Canada. Environ. Res. 2020, 191, 110100. [CrossRef] [PubMed]

38. Morrison, C.N.; Byrnes, H.F.; Miller, B.A.; Kaner, E.; Wiehe, S.E.; Ponicki, W.R.; Wiebe, D.J. Assessing individuals' exposure to environmental conditions using residence-based measures, activity location-based measures, and activity path-based measures. Epidemiology 2019, 30, 166. [CrossRef]

39. Wang, J.; Kwan, M.P.; Chai, Y. An innovative context-based crystal-growth activity space method for environmental exposure assessment: A study using GIS and GPS trajectory data collected in Chicago. Int. J. Environ. Res. Public Health 2018, 15, 703. [CrossRef]

40. Zhao, P.; Liu, X.; Shi, W.; Jia, T.; Li, W.; Chen, M. An empirical study on the intra-urban goods movement patterns using logistics big data. Int. J. Geogr. Inf. Sci. 2020, 34, 1089-1116. [CrossRef]

41. Torres Ochaita, Á. Betados/Vector_2d. GitHub. 2018. Available online: https://github.com/betados/vector_2d (accessed on 26 April 2020).

42. Blundell, R.; Costa Dias, M.; Joyce, R.; Xu, X. COVID-19 and Inequalities. Fiscal Studies 2020, 41, 291-319. [CrossRef]

43. Che, L.; Du, H.; Chan, K.W. Unequal pain: A sketch of the impact of the COVID-19 pandemic on migrants' employment in China. Eurasian Geogr. Econ. 2020, 61, 448-463. [CrossRef]

44. Millett, G.A.; Jones, A.T.; Benkeser, D.; Baral, S.; Mercer, L.; Beyrer, C.; Honermann, B.; Lankiewicz, E.; Mena, L.; Crowley, J.S. Assessing differential impacts of COVID-19 on black communities. Ann. Epidemiol. 2020, 47, 37-44. [CrossRef] [PubMed]

45. Kwan, M.-P. The uncertain geographic context problem. Ann. Assoc. Am. Geogr. 2012, 102, 958-968. [CrossRef]

46. Zhao, P.; Kwan, M.P.; Zhou, S. The uncertain geographic context problem in the analysis of the relationships between obesity and the built environment in Guangzhou. Int. J. Environ. Res. Public Health 2018, 15, 308. [CrossRef]

47. Tuan, Y.F. Space and Place: The Perspective of Experience; University of Minnesota Press: Minneapolis, MN, USA, 1977.

48. Guangzhou Municipal Health Commission. Guangzhou Municipal Health Commission Website-Epidemic Notification Guangzhou Municipal Health Commission. Available online: http://wjw.gz.gov.cn/ztzl/xxfyyqfk/yqtb/index.html (accessed on 10 March 2021).

49. Thakar, V. Unfolding events in space and time: Geospatial insights into COVID-19 diffusion in Washington State during the initial stage of the outbreak. ISPRS Int. J. Geo-Inf. 2020, 9, 382. [CrossRef]

50. Lbs.Amap.Com. AMap Geocoding/Reverse Geocoding API Documentation. Available online: https://lbs.amap.com/api/ webservice/guide/api/georegeo (accessed on 11 August 2020).

51. Geopandas Developers. Geopandas/Geopandas: v0.8.1. Zenodo. 2020. Available online: https://zenodo.org/record/3946761\# .YgeXjpaxVhF (accessed on 11 August 2020).

52. Toms, S. ArcPy and ArcGIS-Geospatial Analysis with Python; Packt Publishing Ltd.: Birmingham, UK, 2015.

53. Gdal/Ogr Contributors. GDAL/OGR Geospatial Data Abstraction Software Library; Open Source Geospatial Foundation: Beaverton, OR, USA, 2021.

54. Lee, H.; Miller, V.J. The disproportionate impact of COVID-19 on minority groups: A social justice concern. J. Gerontol. Soc. Work. 2020, 63, 580-584. [CrossRef] 